Voix et Images

\title{
L'Institution du littéraire au Québec de Lucie Robert
}

\section{David M. Hayne}

Volume 16, numéro 1 (46), automne 1990

Les correspondants littéraires d'Alfred DesRochers

URI : https://id.erudit.org/iderudit/200884ar

DOI : https://doi.org/10.7202/200884ar

Aller au sommaire du numéro

Éditeur(s)

Université du Québec à Montréal

ISSN

0318-9201 (imprimé)

1705-933X (numérique)

Découvrir la revue

Citer cet article

Hayne, D. M. (1990). L'Institution du littéraire au Québec de Lucie Robert. Voix et Images, 16(1), 157-161. https://doi.org/10.7202/200884ar d'utilisation que vous pouvez consulter en ligne.

https://apropos.erudit.org/fr/usagers/politique-dutilisation/ 


\section{L'Institution du littéraire au Québec de Lucie Robert}

\section{par David M. Hayne, Université de Toronto}

Toute nouvelle lecture implique ou exige une relecture: c'est ainsi que les lecteurs qui abordent la thèse de Lucie Robert, l'Institution du littéraire au Québec ${ }^{1}$, auront intérêt à se rappeler l'ouvrage collectif publié en 1986 sous la direction de Maurice Lemire, l'Institution littéraire. Ce rapprochement s'impose pour plusieurs raisons.

D'abord, à cause de la présence, en tête du recueil, d'une communication signée par Lucie Robert et présentée lors de la rédaction de la thèse en question. L'auteure y soulève plusieurs questions méthodologiques et explique certaines décisions prises en cours de route.

Ensuite, pour éclairer le choix du titre de la thèse. Depuis la publication en 1978 de l'ouvrage célèbre de Jacques Dubois, 'le concept d'une "institution de la littérature" est devenu le point de mire de nombreux historiens littéraires qui ont attribué au mot "institution" un sens exclusivement perfectif: pour la plupart d'entre eux, l'institution de la littérature est un ensemble de structures et de normes qui déterminent ou qui influencent la production littéraire d'une société donnée. Pourtant le mot d'institution a un sens premier et dynamique que madame Robert a cru bon de lui restaurer. Il s'agit chez elle, non pas d'une institution-objet, mais d'un processus selon lequel une catégorie culturelle dite "littéraire» est progressivement reconnue par la société, organisée, et insérée dans la réalité de tous les jours.

Finalement, le rappel de l'ouvrage de Maurice Lemire permet d'apprécier toute la nouveauté de l'étude de Lucie Robert, laquelle subordonne la dimension matérielle de l'analyse institutionnelle à sa dimension régulatrice ou normative, répondant ainsi à l'appel formulé lors du précédent colloque par Benoît Melançon (l'Institution littéraire, p. 29-32) dans la continuation des travaux du regretté André Belleau.

Ayant examiné à fond les manuels de littérature québécoise et en particulier ceux de $\mathrm{Mgr}^{\mathrm{gr}}$ Camille Roy, l'auteure se dit convaincue

qu'il n'existe aucun lien nécessaire entre le texte et la littérature et que le corpus littéraire, tel qu'il est constitué dans les manuels d'histoire littéraire par exemple, est le résultat d'une opération de sélection, de valorisation et de canonisation du texte. (p. 3-4) 
Cette prise de position relativiste, qui fait du littéraire non pas une donnée essentielle du texte, mais une valeur transitive, accordée au texte de l'extérieur (p. 4), sert de point de départ à l'enquête. L'auteure se donne pour tâche d'analyser les multiples crises et transformations que subissent la société et ses écrits au cours du processus de socialisation de la littérature. Dans une introduction un peu longue, elle passe en revue ses volumineuses lectures préliminaires, signale les limites qu'elle a dû imposer à ses investigations et se propose d'exploiter un corpus de textes peu connus, rassemblés pendant la préparation du Dictionnaire des cuvres littéraires du Québec. L'utilisation de cette documentation neuve et pertinente, étudiée à la lumière d'une solide réflexion théorique, constitue l'un des aspects les plus originaux de cette recherche inédite.

Le premier chapitre ( Fondations: clôture et socialisation du texte ", p. 33-84) commence par distinguer entre l'écriture, libre et individualisée, et la rédaction, dirigée et encadrée. Les textes qui résultent de l'écriture sont reconnus comme littéraires et ils sont publiés: le texte devient livre, donc marchandise. Avant 1764, le livre était une marchandise étrangère au Québec, produite en Europe et importée. Avec l'introduction de l'imprimerie et la fondation des premières librairies, le livre devint d'abord un objet de commerce, puis un produit artisanal, et enfin un article fabriqué industriellement. Vers 1870 , les ouvrages publiés auparavant dans un périodique commençaient à paraître en volume, initiative encouragée par l'entente signée avec l'abbé $H$.-R. Casgrain pour le choix des livres de prix à distribuer dans les écoles. Entre-temps, les auteurs et les éditeurs se disputaient la propriété de la nouvelle marchandise du livre, querelle qui ne fut réglée qu'avec la promulgation de la Loi canadienne sur le droit d'auteur en 1921. La résolution du problème ne fut aucunement définitive, pourtant, car au XXe siècle tout changea de nouveau: le rôle de l'éditeur se compliqua (publicité, réseaux de distribution, gros tirages, prix avantageux) et les auteurs, plus agressifs, se réunirent en association afin d'appuyer leurs revendications collectives.

Le deuxième chapitre ("Questions de stratégie: la double condensation du pouvoir", p. 85-185) étudie les relations, harmonieuses ou conflictuelles, entre les secteurs public et privé, entre l'État et la société civile. Dans les années 1830 , le sentiment patriotique favorisait la création d'une littérature nationale: un public de lecteurs instruits se forma, et l'État y concourut en adoptant les premières lois scolaires et en subventionnant des bibliothèques municipales et paroissiales. La réaction de l'Église se fit sentir à partir de 1840: elle fonda ses propres journaux, créa l'Euvre des bons livres, organisa des salles de lecture, multiplia les collèges classiques, et lutta pour l'abolition du ministère de l'Instruction 
publique. Le clergé exerçait un droit de censure et contrôlait la distribution des livres; il intervint directement dans la production littéraire lors du mouvement de 1860, et encore plus activement au moyen de la création d'éditions catholiques vers la fin du siècle. Cette action apostolique prit plus d'ampleur au XXe siècle avec la formation de syndicats catholiques et du mouvement d'Action sociale catholique, avec l'encouragement accordé au régionalisme littéraire, et avec l'organisation des trois grands congrès de la langue française en 1912,1937 et 1952 . Toutes ces initiatives facilitaient par ailleurs l'assimilation de nouvelles catégories de lecteurs: les jeunes, les femmes et les classes populaires.

La guerre de 1939-1945 communiqua à l'édition canadiennefrançaise une impulsion sans précédent, alors que les maisons canadiennes recevaient l'autorisation de rééditer des ouvrages français bloqués par l'occupation allemande de la France. Cette expansion fut de courte durée cependant, et en face des démarches agressives de l'édition française après la libération, il fallait penser à assurer une industrie nationale du livre. Le rapport Massey de 1951 constitue le premier énoncé de politique globale dans le domaine culturel, si l'on excepte les programmes énoncés par le clergé ou par les congrès de la langue française (p. 139). Pourtant l'intervention de l'État canadien en faveur d'une politique culturelle pancanadienne exclut l'autonomie culturelle du Québec: celle-ci deviendrait l'une des principales préoccupations de la Révolution tranquille des années soixante.

Le troisième chapitre ("Lillusion juridique: le fétichisme de la littérature ", p. 147-216) s'ouvre sur une analyse des deux concepts de l'institution, l'un basé sur le consentement et l'unanimité relative des individus qui la composent, l'autre fondé sur la notion de discipline et de contrainte exercées par un "appareil» quelconque. C'est ici que l'auteure fait un retour dans le passé afin de brosser à grands traits l'histoire des conditions d'apparition $d u$ savoir moderne [...] en Europe (p. 161). S'inspirant de l'ouvrage d'Alain Viala, elle rappelle le rôle des académies au XVII ${ }^{\mathbf{e}}$ siècle et propose un parallèle entre les académies humanistes et les parlements, lesquels partageaient une même fonction de conseil auprès du souverain et un même caractère juridique. Au Québec, les premières sociétés littéraires et scientifiques s'organisaient au début du XIX ${ }^{\mathbf{e}}$ siècle; bilingues et orientées surtout vers les sciences, elles furent graduellement remplacées après 1830 par des sociétés francophones consacrées à la littérature et à l'histoire nationales. L'académisme de ces sociétés privilégiait l'écrit et les belles-lettres, tandis qu'il folklorisait l'oral et la culture populaire: Le goût, français et universel, déterminait la forme; le fond serait canadien, mais d'un canadianisme filtré et idéalisé. La réflexion sur "l'âme" nationale se poursuivit au tournant du siècle 
dans les travaux des premiers sociologues et économistes (Léon Gérin, Edmond de Nevers, Errol Bouchette) et dans les études des critiques littéraires (Camille Roy, Émile Chartier), car la critique littéraire [faisait] à cette époque partie des sciences humaines (p. 186). Les manuels de Mgr Roy assuraient l'axiomatisation, ou l'acceptation aveugle et non critique, d'un certain corpus historique de littérature canadienne-française.

Le troisième chapitre se termine par quelques pages consacrées à la modernisation du Québec et à la modernité littéraire. La modernisation serait la préoccupation de la génération perdue née vers 1900 (Marcel Dugas, Claude-Henri Grignon, Berthelot Brunet et, curieusement, Louis Dantin, né en 1865!), dont l'audace trouvait son pendant dans l'esprit d'indépendance d'une génération de femmes cultivées, nées vers la même date (Jovette Bernier, Simone Routier, Éva Senécal et Alice Lemieux). Une autre génération, celle de la crise, a flirté aussi bien avec le fascisme qu'avec le socialisme (p. 201), mais elle a doté le Québec de plusieurs organismes et périodiques (la Société des écrivains canadiens-français, la Relève, le Jour) dont l'influence serait décisive. L'année 1934 marqua une rupture et le climat intellectuel changea de façon irréversible. Les sciences sociales furent finalement acceptées par l'université, et en 1954, un article retentissant de Jeanne Lapointe signala l'émancipation de la critique littéraire.

Un simple résumé de ces grandes lignes ne suffit pas à donner une idée juste de l'étonnante richesse de l'ouvrage de madame Robert. Celui-ci contient des dizaines de remarques perspicaces et suggestives, par exemple, sur le rôle du clergé en tant qu'importateurs de publications étrangères au XIX ${ }^{e}$ siècle (p. 109), sur la multiplication des enquêtes littéraires dans les années 1930 (p. 203) ou bien sur la folklorisation actuelle des études de littérature québécoise (p. 215). En revanche, certains événements que l'on aurait crus significatifs, tels que les concours de poésie de l'université Laval (p. 181), le projet de réforme de la loi scolaire en 1897-1898, le rapport "explosif" de la Commission Bouchard (p. 225), le Bill 60 ou la création des cégeps sont expédiés rapidement ou passés sous silence. Il aurait été intéressant aussi de trouver ici l'historique des désignations successives de la "littérature" au Québec: canadienne, canadienne-française, française du Québec, québécoise, et ainsi de suite.

Constatons enfin que l'Institution du littéraire au Québec n'est pas un livre qu'on lit à la plage ou debout dans le métro. Non linéaire dans sa structure, non chronologique dans son développement, débordante d'allusions interdisciplinaires, abstraite dans son lexique et minutieuse dans ses distinctions, la thèse de Lucie Robert, comme tous les ouvrages innovateurs, exige de son lecteur ou de sa 
lectrice une attention soutenue et la patience de revenir sur les passages difficiles. Pourtant, s'il m'est permis d'emprunter une image à un autre domaine de spécialisation de notre auteure, le jeu vaut bien la chandelle.

1 Lucie Robert, l'Institution du littéraire au Québec, Québec, les Presses de l'université Laval, 1989, 272 p. (CRELIQ. Vie des lettres québécoises, 28). 